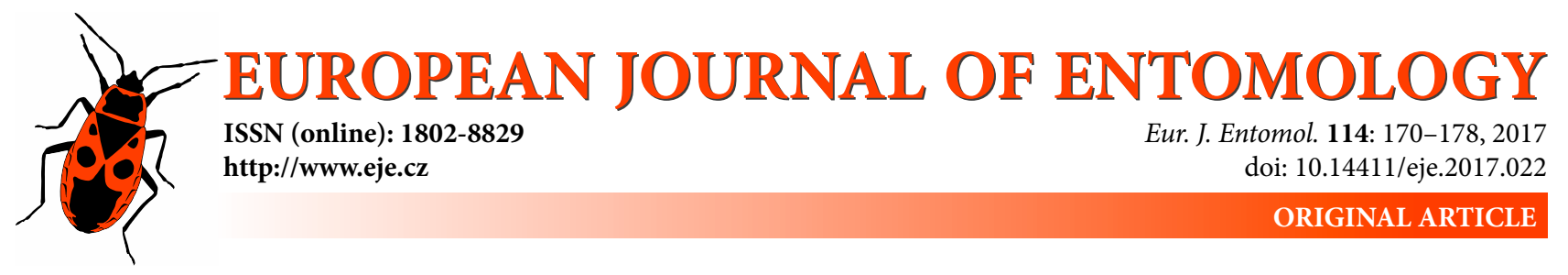

\title{
Double strand RNA-mediated RNA interference through feeding in larval gypsy moth, Lymantria dispar (Lepidoptera: Erebidae)
}

\author{
SaIKat Kumar B. GHOSH and Dawn E. GUNDERSEN-RINDAL* \\ United States Department of Agriculture - ARS Invasive Insect Biocontrol and Behavior Laboratory, Beltsville, Maryland, USA; \\ e-mails: saikat.ghosh@ars.usda.gov, dawn.gundersen-rindal@ars.usda.gov
}

Key words. Lepidoptera, Erebidae, Lymantria dispar, gypsy moth larvae, RNAi, dsRNA, ingestion

\begin{abstract}
RNA interference (RNAi) technology uses dsRNAs to silence specific targeted genes by downregulating their expression. It has become a potent tool for functional and regulatory studies of insect genes and has potential to be applied for insect control. Though it has been challenging to generate effective RNAi in lepidopteran insects, in the current study this technology was applied to develop specific RNAi-based molecular tools that could be used to negatively impact the invasive lepidopteran forest pest, gypsy moth (GM). GM midgut-specific genes were selected for dsRNA design from larval transcriptome profiles. Two methods were used to produce specific dsRNAs, bacterial expression and in vitro synthesis, which were then fed per os to GM larvae. Depletion of uncharacterized gene targets known as locus 365 and locus 28365, or their stacked combination, depleted target transcripts in a sequence specific manner and resulted in $60 \%$ reduction in body mass. Treated GM females that were able to moult to the adult stage displayed an approximately two-fold reduction in egg masses. These have potential to be developed as molecular biopesticides for GM.
\end{abstract}

\section{INTRODUCTION}

Lymantria dispar, the gypsy moth (GM), is a serious insect pest of the North American forests where larvae feed on over 300 tree and shrub species, especially hardwood and shade trees. Measures to control the pest and reduce its spread have been implemented in the United States, including augmentative release of natural enemies, chemical pesticides, mating disruption using a chemical pheromone, and use of microbial biopesticides [nucleopolyhedrovirus or Bacillus thuringiensis (Bt)] (Höfte \& Whiteley, 1989). These measures have been employed with variable success and resistance to biopesticides has evolved rapidly in larval populations. Recent analyses of GM-associated genes generated through characterization of transcriptomes of both an GM-derived cell line IPLB-Ld652Y (Sparks \& Gundersen-Rindal, 2011) and the GM larval midgut (Sparks et al., 2013), identified genes with potential utility for developing molecule-based targeted approaches for GM control.

The discovery of RNA mediated interference (RNAi) has facilitated research to understand gene function and regulation. RNAi provides an efficient silencing technique to deplete targeted genes by degradation of mRNA into short RNAs (Bernstein et al., 2001; Ketting et al., 2001; Martinez et al., 2002; Bartel, 2004). The major limiting factors for in vivo application of dsRNA-mediated RNAi have been the cellular uptake of dsRNA, delivery method, and stability of dsRNA after its delivery (Yu et al., 2012). Effective approaches for RNAi delivery - such as injecting, soaking or feeding the extracellular RNA - have been reported with varied success. Fire and colleagues first observed the phenomenon of RNAi by injecting and feeding dsRNA in the nematode, Caenorhabditis elegans to deplete the unc-22 gene generating twitching phenotypes (Fire et al., 1998; Timmons \& Fire, 1998). Subsequently, RNAi was successfully performed in other animals such as Drosophila melanogaster to downregulate the frizzled and frizzled 2 genes (Kennerdell \& Carthew, 1998). Clemens and colleagues, used RNAi in Drosophila S2 tissue cultures cells to inhibit expression of the DSOR1 (mitogenactivated protein kinase kinase, MAPKK) which prevented the activation of the downstream ERK-A (MAPK) in the insulin signal transduction pathway and vise versa (Clemens et al., 2000).

Though lepidoteran insects have been somewhat resistant to RNAi, some successful dsRNA-mediated RNAi instances have been observed (Terenius et al., 2011). In one instance, dsRNA for the aminopeptidase gene (slapn) injected into the midgut of Spodoptera litura reduced its expression and susceptibility to a $B t$ cry $1 \mathrm{C}$ protein (Rajagopal et al., 2002). Other studies demonstrated RNAi

\footnotetext{
* Corresponding author; e-mail: dawn.gundersen-rindal@ars.usda.gov
} 
by feeding dsRNA either in a sucrose droplet to reduce the transcription of carboxylesterase gene (EposCXE1) in Epiphyas postvittana or from plants engineered to produce dsRNA against cytochrome $\mathrm{P} 450$ gene (CYP6AE14) in Helicoverpa armigera (Turner et al., 2006; Mao et al., 2007).

For use of dsRNAs in lepidopteran insect biocontrol, elements including (a) appropriate gene targets, (b) effective RNAi causing negative impact, and (c) a delivery method through feeding are all needed to make their use in the environment practical. In the current study, specifically designed dsRNAs were examined for efficiency in gene knockdown, depletion, and effects on GM larvae. These specific dsRNAs were produced by (i) bacterial expression or (ii) in vitro synthesis methods and were delivered to larvae per os by feeding.

\section{MATERIALS AND METHODS}

\section{Gypsy moth rearing}

GM larvae were reared from single egg masses to reduce variability. GM eggs were acquired from USDA/APHIS, Otis ANGB, MA. Larvae were reared to the third instar stage on artificial diet (AD) consisting of wheat germ, USDA vitamin mix, casein, wesson salts, sorbic acid, methyl paraben, and agar (Bell et al., p. 599 in Doane \& McManus, 1981) in $180 \mathrm{ml}$ plastic cups. Larvae were staged by rearing in groups of approximately 50 individuals until head capsule formation was observed signifying entry into the larval molt to 3 rd instar.

\section{Cloning and dsRNA expression in E. coli HT115(DE3)}

GM-specific gene regions were identified using NCBI BLASTx to eliminate off target insect homologies, examining GM transcriptomic profiles (Sparks \& Gundersen-Rindal, 2011; Sparks et al., 2013), and selecting regions sized between 200-500 base pairs. PCR products were generated from genomic DNA using oligonucleotide primers (Table S2) and cloned into the multiple cloning site (MCS) of the L4440 plasmid (Addgene plasmid 1654) (Timmons et al., 2001) using SmaI and PmlI restriction sites to yield $A P N 1$, locus 365, locus 28365, Osi 9 and locus 4413 constructs. Subsequently, each plasmid was transformed into $E$. coli strain HT115(DE3) [Caenorhabditis Genetics Center at the University of Minnesota (Timmons et al., 2001)] and plated on ampicillin and tetracyclin containing NZCYM-agar plates.

An overnight culture of transformed HT115(DE3) + plasmid was used to inoculate a $500 \mathrm{ml} \mathrm{NZCYM}$ media to an $\mathrm{OD}_{600}=0.05$ and grown to an $\mathrm{OD}_{600}=0.4$. Cells were induced with IsopropylB-D-1-thiogalactopyranoside (IPTG) to a final concentration of $0.4 \mathrm{mM}$ and further grown for $4 \mathrm{~h}$ at $37^{\circ} \mathrm{C}$. Cells were harvested by centrifugation, weighed and stored at $-80^{\circ} \mathrm{C}$.

\section{In vitro synthesis of double stranded RNA}

The above PCR products were re-amplified using primers flanked with a T7 promoter sequence (5'-GAA TTA ATA CGA CTC ACT ATA GGG AGA-3'). LacZ (mock) fragment was amplified from the E. coli genomic DNA as a control (primers listed in Table S2). PCR-amplified DNA was purified using the QIAquick PCR purification kit (Qiagen, Germantown, MD). In vitro transcription to yield dsRNA was performed either by using the Megascript RNAi kit (Life Technologies, Carlsbad, CA) or T7 RNA polymerase by the manufacturer's protocol (Promega, Madison, WI). This reaction was incubated overnight at $37^{\circ} \mathrm{C}$. Reactions were then centrifuged for $2 \mathrm{~min}$ at 13,000 rpm to pellet the magnesium pyrophosphate. This supernatant was treated with
2 units of RQ1 RNase-free DNase (Promega) at $37^{\circ} \mathrm{C}$ for $30 \mathrm{~min}$, then extracted one time with an equal volume of phenol/chloroform/isoamyl alcohol $(25: 24: 1)$ followed by chloroform alone. The resulting aqueous layer was precipitated in one-fifth-volume ammonium acetate and 3 volumes of $100 \%$ ethanol; washed with $75 \%$ ethanol and dissolved in nuclease free water.

\section{RNAi in gypsy moth}

Two approaches for dsRNA synthesis were investigated for inducing RNAi in GM larvae: (i) expression by HT115(DE3) bacteria and (ii) synthesis by in vitro transcription. Both were then mixed with the appropriate ADs and fed to larvae.

A freeze-dried $\mathrm{AD}$ pellet was powdered and placed in the well of a plastic bioassay tray (Bio-BA 128; BioServ, Frenchtown, NJ). The diet was rehydrated by applying $300 \mu \mathrm{l}$ of dsRNA-induced bacterial culture and green food coloring to monitor ingestion and excretion (Martin, 2004). For in vitro synthesized dsRNA, $24 \mathrm{mg}$ of the freeze-dried AD was rehydrated using $100 \mu \mathrm{l}$ of $0.2 \mu \mathrm{g} /$ $\mu 1$ of dsRNA containing $2 \%$ PEG 8000 . Third instar larvae were starved for $24 \mathrm{~h}$ and placed on each pellet, a total of 3 larvae per assay. Controls consisting of empty vector (L4440), mock control ( $\mathrm{LacZ}$ dsRNA) and water only were also tested. Larvae were placed at $27^{\circ} \mathrm{C}$ until they reached the adult stage. During this period total body mass and egg masses of the females reaching the adult stage were monitored for phenotypic effects of RNAi. To study the level of transcripts by qPCR, the larvae were fed on dsRNA continuously for 5 days with replenishment after 3 days.

\section{Synthesis of cDNA for transcript level measurements}

GM gut tissue was isolated by dissection subsequent to dsRNA treatment for cDNA synthesis. Total RNA was isolated from the tissue using either TRIzol (Invitrogen, Carlsbad, CA, USA) or the RNeasy kit (Qiagen). Reverse transcriptase PCR was used to generate cDNA from 200 ng of total RNA using Superscript Reverse Transcriptase III (Life Technologies).

\section{Quantitative real-time PCR analysis}

Transcript expression levels were measured by quantitative realtime PCR (qPCR) (primers in Table S2) using SensiMix SYBR from Bioline (Taunton, MA, USA). Data were analyzed with ABI Prism sequence detection system software (Waltham, MA, USA). All analyses were performed in the linear range of amplification. The data was plotted using Kaleidagraph (Synergy software, Reading, PA, USA). The height of each box represented the mean average of sample-specific $2^{-\Delta \Delta C}{ }_{T}$ values. The samples were then normalized to $18 \mathrm{~S}$ RNA expression that remained unchanged upon dsRNA depletion of the targeted genes. Data was expressed as mean \pm SEM from at least three independent experiments. Statistical analysis was performed using one-way ANOVA analysis.

\section{RESULTS}

\section{Gene selection and analysis of dsRNA induced in bacteria}

Systemic RNAi transmitted through bacterial expression of dsRNA is commonly used in C. elegans (Timmons $\&$ Fire, 1998; Kamath et al., 2001). Only genes that were strictly specific to GM were selected from the transcriptome profiles (Sparks et al., 2013). Of a total of 10 genes identified, five that readily amplified from genomic DNA were selected for depletion by RNAi (Table S1). These were: aminopeptidase N1 (APN1) (locus 22), identified as the CrylAc endotoxin of $B t$ receptor in GM (Garner et al., 1999); Osiris 9 (Osi9) (locus 5421), a gene of unknown 

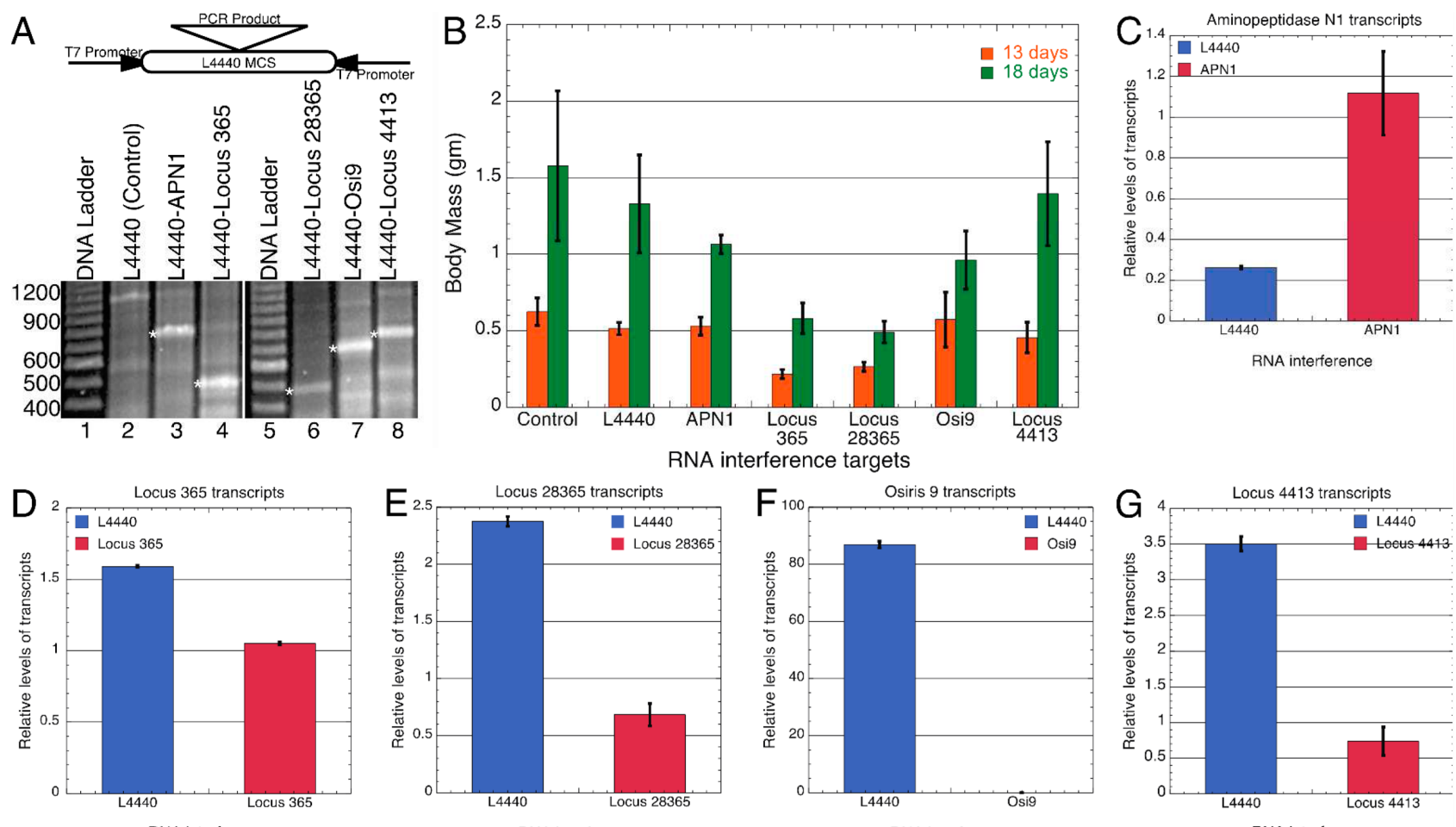

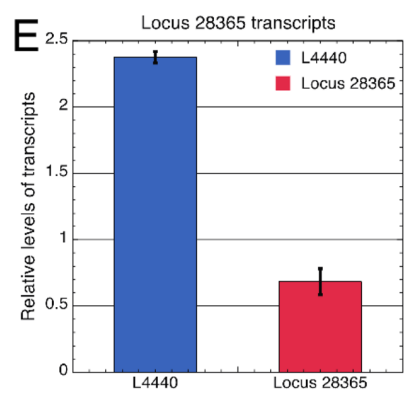

RNA interference

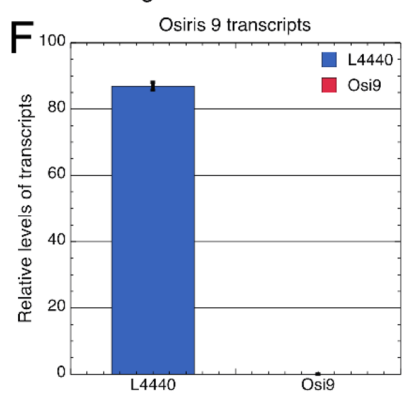

RNA interference

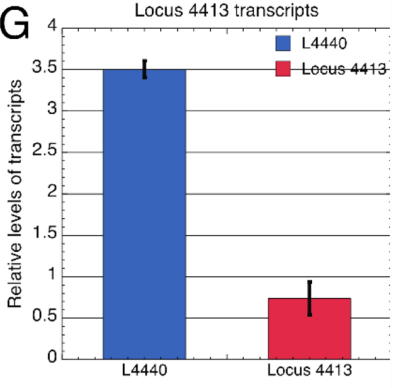

RNA interference

Fig. 1. RNAi using bacterially expressed dsRNA. A- total RNA isolated from $2 \mathrm{ml}$ of induced bacterial culture using TRIzol and treated with RQ1 DNase and RNaseA. Resistant bands denoted by (*) indicate dsRNAs for gene specific constructs analyzed by $1 \%$ AGE (Agarose Gel Electrophoresis). B - third instar larvae were fed on bacterially expressed dsRNA as indicated. Body mass was measured on the $13^{\text {th }}$ $(P=0.0519)$ and $18^{\text {th }}(P=0.0936)$ days after stopping dsRNA feeding $(n=3$, error bars indicate SEM; statistical analysis was performed using one-way ANOVA analysis with Fisher's LSD post hoc test shown in Table S3A). C-G - expression of (C) APN1 ( $P=0.0143)$, (D) locus $365(P<0.0001)$, (E) locus $28365(P<0.0001)$, (F) Osi9 $(P<0.0001)$, and $(\mathrm{G})$ locus $4413(P=0.0002)$; genes in the mid-gut of $\mathrm{GM}$ were analyzed using APCR. (Three biological replicates, error bars indicate SEM; One-way ANOVA $P<0.0001$ ). Statistical analysis for $C$ to $\mathrm{G}$ was performed using One-way ANOVA with Fisher's LSD post hoc test.

function that is syntenic between B. mori and Drosophila (Shah et al., 2012; Suetsugu et al., 2013) and displayed homology to the transcriptome profile of GM; genes known only as locus 365, a hypothetical protein in Pectinophora gossypiella, locus 28365 unique to the GM transcriptome; and locus 4413, a predicted protein in Nematostella vectensis with partial homology to vitellogenin an egg yolk precursor protein in insect females (Hagedorn \& Kunkel, 1979) in Rhipicephalus microplus.

To confirm synthesis of dsRNA in HT115(DE3), a part of the induced culture was analyzed and dsRNA expression observed for the selected fragments (Fig. 1A). The observed bands of expected size were insensitive to RNase $\mathrm{A}$ and DNase treatments and hence were assumed to be dsRNA. There was a 163 bp difference in size between the cloned fragments and the induced dsRNA contributed by the MCS region of L4440 vector, evident from the mobility of the dsRNA bands (Fig. 1A, lanes 3, 4, 6, 7 and 8). To avoid any phenotypes resulting from the L4440 plasmid vector, we used the empty vector as a control (Fig. 1A, lane 2).

\section{Bacterially expressed dsRNA stimulated RNAi in gypsy moth larvae}

RNAi was most effective when larvae were allowed to feed continuously on extracellular dsRNA (Timmons et al., 2001; Li et al., 2011). To test the effects of RNAi, bacterial suspension containing induced dsRNA mixed with
$\mathrm{AD}$ was fed continuously to the $3^{\text {rd }}$ instar larvae for 5 days and transferred to AD devoid of dsRNA. Observations indicated that the larvae fed with locus 365 and locus 28365 dsRNA showed a striking two-fold loss in body mass after 13 and 18 days post-feeding of dsRNA when compared to the control (Fig. 1B and Table S3A). A significant two-fold decrease in body mass was observed in larvae treated with locus 365 dsRNA after 13 days and a similar decrease was also observed in larvae that were treated with either locus 365 or locus 28365 dsRNAs 18 days post-feeding of dsRNAs compared to L4440 control (Fig. 1B and Table S3A). This decrease in body mass may be symptomatic of decreased dietary intake due to RNAi.

As an alternative test of dsRNA mediated RNAi, the levels of transcript were evaluated in the gut of a subset of the larvae subsequent to dsRNA ingestion by qPCR. The expression of loci 365, 28365, 4413 and $O s i 9$ were significantly reduced (Fig. 1D-F). Interestingly, APN1 showed a substantial 5-fold increase in level of transcripts (Fig. 1C). This was most likely an anti-bacterial response to the $E$. coli bacteria fed to the larvae. A less likely possibility could be the gene may be part of a feedback loop controlling its own expression. At a threshold of translational blockage the animal may be compensating by up-regulating gene transcription resulting in enhanced mRNA expression as observed in the case of the CREB1-positive feedback loop (Liu et al., 2008). In particular these results conclude that 


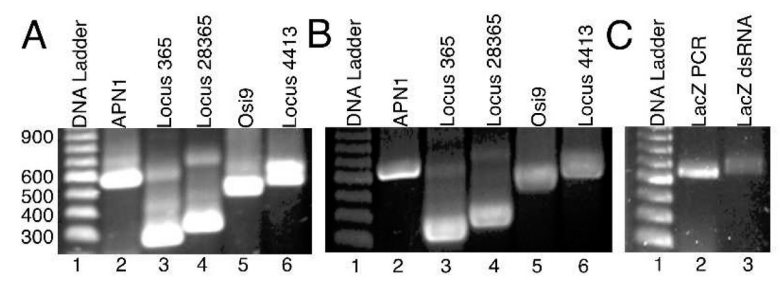

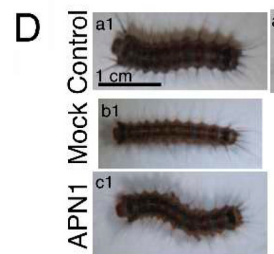
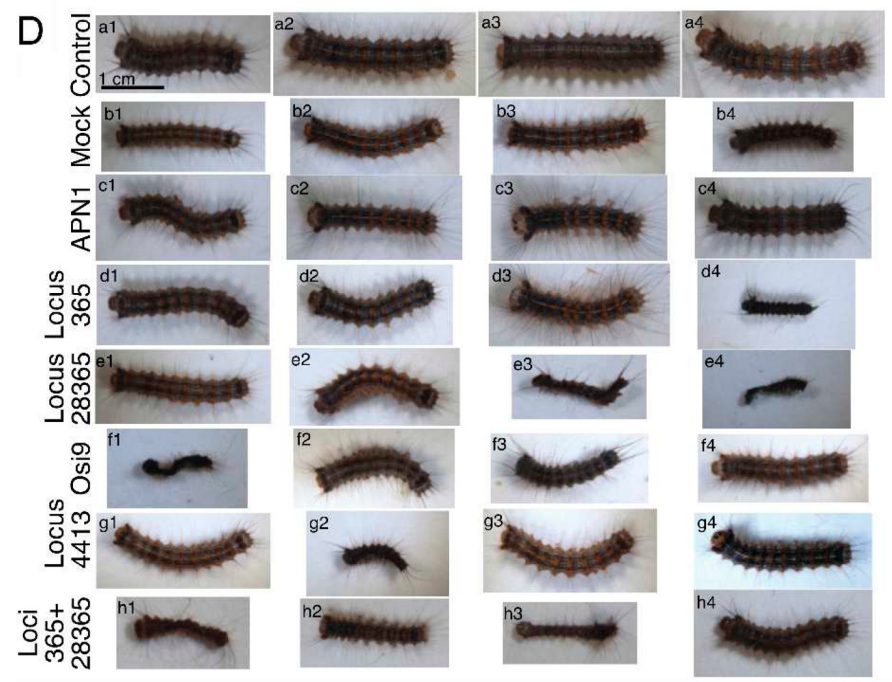
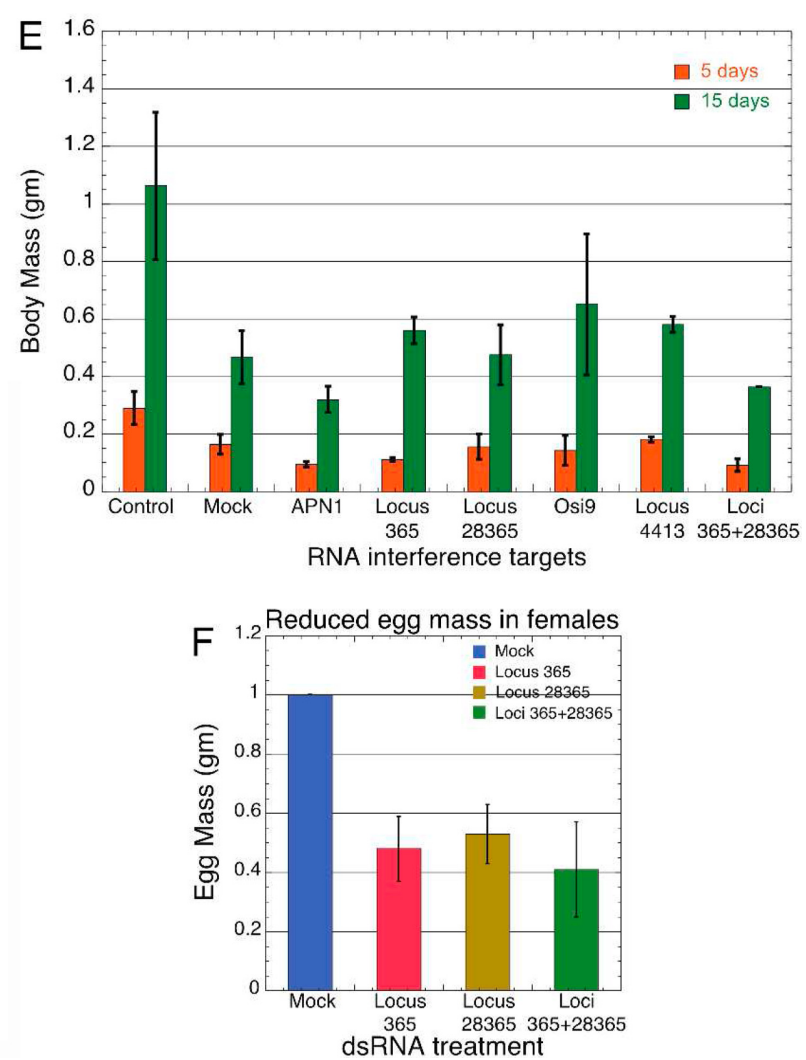

Fig. 2. In vitro transcribed dsRNA mediated RNAi. A - PCR fragments of the indicated genes amplified from PCR products from genomic DNA using primers containing T7 promoter sequence. B - in vitro transcribed dsRNA from indicated specific-genes. C - LacZ gene PCR product and in vitro transcribed dsRNA. All nucleic acids were analyzed by $1 \%$ AGE (Agarose Gel Electrophoresis). D - third instar larvae were fed on in vitro transcribed dsRNA indicated as LacZ RNAi (Mock), APN1, locus 365, locus 28365, Osi9, locus 4413, stacked loci 365 $+28365($ Scale $=1 \mathrm{~cm})$. AD was mixed with water as control. E - effect of RNAi on body mass measured on days $5(P=0.02897)$ and 15 $(P=0.00146)$ after stopping dsRNA feeding. (Four biological replicates, error bars indicate variations in biological samples; all statistical analysis was performed using One-way ANOVA with Fisher's LSD post hoc test shown in Table S3B). F - reduction in the egg masses in females post dsRNA treatment, $P=0.03578$, Fisher's LSD for Mock vs locus $365, P=0.0497$; Mock vs locus $28365, P=0.00138$; and Mock vs locus $365, P=0.0083$. (Three biological replicates, error bars indicate variations in biological samples; all statistical analysis was performed using One-way ANOVA with Fisher's LSD post hoc test).

RNAi may be achieved by feeding bacterially expressed dsRNA in GM.

\section{In vitro dsRNA synthesis for feeding}

A second objective of this study was to develop sustainable methods for delivery of dsRNA for biocontrol of pests. Using $B t$ as pesticide is a common practice but using bacterial culture as a large-scale delivery system is unfeasible in the environment. Moreover, a previous study reported L4440 empty vector may induce gene silencing (Grishok et al., 2005) although we did not observe this occurrence. We investigated the possibility of using in vitro synthesized dsRNA for RNAi through feeding, which has never been assessed in GM. T7 promoter-flanked DNA fragments (Fig. $2 \mathrm{~A}$, lanes 2-6) were in vitro transcribed to generate their respective dsRNAs (Fig. 2B, lanes 2-6) and $\operatorname{LacZ}(2 \mathrm{C})$ as control. Slower mobility of dsRNA was observed which is common when comparing dsRNA to dsDNA (Livshits et al., 1990). The dsRNA was observed to be stable over the period of feeding.

\section{dsRNA mediated RNAi contributed to physical variations in gypsy moth}

We investigated the long-term effect of RNAi in GM by allowing larvae to feed on in vitro synthesized dsRNA and noting any phenotypic changes. We noticed that control larvae were physically better developed when compared to mock treated (Fig. 2D, compare panels a1-a4 to b1-b4). However, both control- and mock-treated larvae developed to be healthy adults. RNAi caused mortality in larvae treated with dsRNA against locus 28365 (Fig. 2D, panel e4) and Osi9 (Fig. 2D, panel f1) after 2 days of treatment. More mortality was observed in larvae treated with dsRNA for locus 365 (Fig. 2D, panel d4), locus 28365 (Fig. 2D, panel e3), locus 4413 (Fig. 2D, panel g2) and loci $365+28365$ (Fig. 2D, panels h1 and h3) after 4 days of stopping dsRNA feeding. While, Osi9 and loci $365+28365$ dsRNA caused mortality after 10 days of stopping per os feeding (Fig. 2D, panels $\mathrm{f} 4$ and h2). Body mass of these larvae at $5^{\text {th }}$ - and $15^{\text {th }}$ day stages post dsRNA treatment was also measured. Larvae treated with $A P N 1$, locus 365 , locus 28365 , Osi9, locus 4413 and stacked loci $365+28365$ dsRNAs displayed a 2to 3 -fold decrease in body mass compared to control after 5 and 15 days (Fig. 2E and Table S3B). A similar depletion was observed only in larvae fed on stacked dsRNA for loci $365+28365$ after 5 days post dsRNA treatment (Fig. 2E and Table S3B). Moreover, RNAi caused mortality except in controls and $A P N 1$ dsRNA treated larvae (Fig. 2E). Next we measured the egg mass from newly emerged adults as 

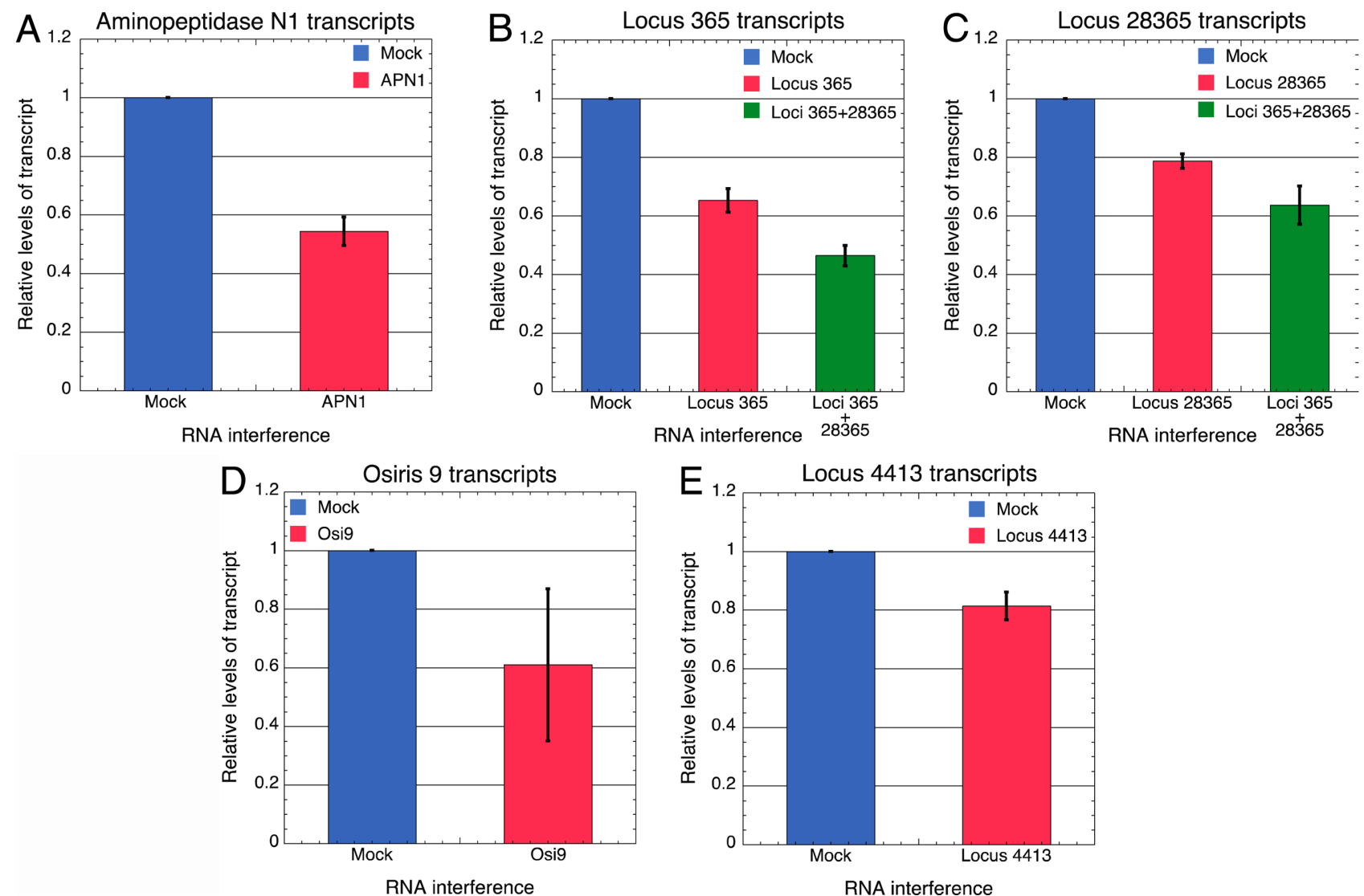

Fig. 3. Quantitative RT-PCR analysis of transcript levels after RNAi. Expression of $(\mathrm{A}) A P N 1(P=0.00632)$, (B) locus $365(P<0.0001)$ (Mock vs locus $365 P=0.0002$; Mock vs loci $365+28365 P<0.0001)$, (C) locus $28365(P=0.0376)$ (Mock vs locus $365 P=0.1157$; Mock vs loci $28365+365 P<0.012)$, (D) Osi9 $(P=0.2885)$, and $(\mathrm{E})$ locus $4413(P=0.1172)$ genes analyzed by qPCR. The stacked dsRNA for loci $365+28365$ with the respective genes for RNAi in (B) and (C) by qPCR. LacZ RNAi (Mock) served as control. (Six biological replicates, error bars indicate SEM, One-way ANOVA $P=0.0077)$. Statistical analysis was performed using One-way ANOVA with Fisher's LSD post hoc test.

a measure of fecundity. Adult female eclosions were observed only in the control (Fig. 2D, panels a1, a3 and a4), mock (Fig. 2D, panel b1) and locus 365 (Fig. 2D, panels $\mathrm{d} 1$ and $\mathrm{d} 2$ ) animals. Locus 365 depleted females had half as many eggs compared with mock-treated animals. In a separate experiment larvae treated with dsRNA for locus 365 and locus 28365 and allowed to develop to the adult stage displayed a $50 \%$ reduction in the egg mass of females compared to the mock treatment. When the dsRNAs for loci 365 and 28365 were stacked, the resulting egg mass was reduced by $60 \%$ than mock treated larvae (Fig. $2 \mathrm{~F}$ ). Although only a modest difference was observed in the body mass of dsRNA treated animals as compared to the mock treated, the two-fold decrease in egg mass indicated that dsRNAs of locus 365, locus 28365 and stacked loci 365 and 28365 effectively induced RNAi in the animals. These results together indicated that RNAi aimed at the gene targets affected both development and fecundity of the animals.

\section{RNAi using in vitro synthesized dsRNA depleted expression of target genes}

If RNAi induces physiological variations then depletion of target gene expression upon dsRNA ingestion should be observed. To test this prediction and whether stacking dsR-
NAs for loci 365 and 28365 in the diet had any potential additive or synergistic effects, levels of transcripts were assayed by qPCR for depletion in expression of target genes. Observations revealed that RNAi against APN1 and locus 365 significantly depleted the level of expression of these transcripts and a biologically relevant decreased level of expression in larvae feeding on locus 28365 (Fig. 3A, B and C). Correspondingly, in the case of stacked dsRNAs, levels of transcripts were significantly lower than for individual RNAi compared to their individual controls (Fig. $3 \mathrm{~B}$ and $\mathrm{C}$ ). We did not notice hyperexpression of APN1 as observed earlier (Fig. 1C), instead there was significant depletion in its expression (Fig. 3A). Cheng and colleagues observed that after infection with microorganisms the expression of genes of the APN family were upregulated in Bombyx mori (Cheng et al., 2016). Our observation corroborates this finding wherein bacterially expressed dsRNA showed overexpression of $A P N 1$ (Fig. 1C) to combat the bacterial pathogen, however, in vitro synthesized dsRNA diminished the level of APN transcripts (Fig. 3A) eliciting an RNAi response in the animal. Thus we conclude that RNAi in GM can be successfully accomplished by feeding in vitro synthesized dsRNA with potential for additive to synergistic effect when two dsRNAs are stacked. 


\section{DISCUSSION}

RNA interference (RNAi) technologies used to silence specific targeted genes by degrading RNAs have great potential to be applied for insect control that is safe and effective. However, induction of robust systemic RNAi in lepidopteran insects has been challenging and depends on the methods of delivery, uptake of the delivered dsRNA and the RNAi core machinery necessary for effective gene knockdown (Terenius et al., 2011). The Argonaute and PPD (PAZ and Piwi domains) family of proteins Ago1, Ago2a, Ago $2 b$ and $A u b$, and endosomal escape of dsRNA are essential for an efficient RNAi in insects (Zamore, 2001; Yoon et al., 2016). Lepidopteran insects have been refractory to RNAi, their RNAi pathway genes or machinery have not yet been fully elucidated, and dsRNAs delivered orally to these chewing insects must pass through the alkaline midgut where they could be damaged. For example, Spodoptera frugiperda and Helicoverpa zea larvae showed enhanced dsRNA degradation and instability in the gut lumen and lack of subsequent processing of ingested plant dsRNAs into the $21 \mathrm{nt}$ siRNAs essential for RNAi (Ivashuta et al., 2015). Similarly, Wang and colleagues, reported that efficiency of chitinase gene depletion in Spodoptera litura was diminished due to enzymatic degradation of dsRNAs in the hemolymph and gut juice (Wang et al., 2016). Thus, use in the environment of specific dsRNAs as biopesticides for lepidopteran larvae will require efficacious oral delivery and gene-specific dsRNA stability.

RNAi has proved to be a potent tool for functional and regulatory studies of insect transcripts. Several successful methods of dsRNA delivery have been described, including microinjection to deliver an effective dose of dsRNA to a precise location in the animal, soaking, and ingestion to induce RNAi (Huvenne \& Smagghe, 2010). For example, dsRNA-mediated depletion of aminopeptidase and vitellogenin receptor genes in S. litura by microinjection has been demonstrated (Rajagopal et al., 2002; Shu et al., 2011). Oral delivery of dsRNA in insects facilitates largescale gene screening by RNAi that aids the development of biopesticides (Kamath et al., 2001; Tian et al., 2009). In the present study, orally delivered dsRNAs for depletion of specific GM target genes were analyzed for assessment of their RNAi-based biomolecular impact on the pest. Previous studies demonstrated that the stability of siRNA was increased when complexed with polyethylene glycol (PEG) which effectively protected siRNAs from hydrolysis by serum nucleases and prevented absorption by serum proteins (Baigude \& Rana, 2009; Draz et al., 2014). Therefore, in the present study, a complex of dsRNAs with $2 \%$ PEG was delivered to larvae to prevent degradation and stabilize dsRNAs in the midgut. Two methods were used for synthesizing dsRNAs, (i) bacterial expression (Timmons \& Fire, 1998), and (ii) in vitro synthesis, for per os delivery to GM larvae.

Sequences homologous to C. elegans systemic RNAi deficient-1 (sid-1) gene responsible for uptake in the gut and the systemic spread of dsRNAs, have been identified in $A$. mellifera and B. mori (Winston et al., 2002; Honeybee Ge- nome Sequencing Consortium, 2006). We observed a similar homologue of $B$. mori Sid-1 in the GM transcriptome (Sparks et al., 2013). Hence, the primary mode of delivering dsRNA per os to the larval midgut to induce systemic RNAi had potential to be effective. Results indicated that GM larvae could orally ingest either bacterially expressed or in vitro synthesized dsRNAs, leading to targeted gene depletion in the midgut. When fed to larvae, dsRNAs for locus 365 and locus 28365 were responsible for consistent depletion of transcripts in vivo, while stacking these two dsRNAs led to an additive effect, with potential for a synergistic effect. Phenotypic analyses of these larvae demonstrated poor development and higher mortality upon depletion of target genes.

Members of a distinctive group of dsRNA nucleases predominantly expressed in insect gut tissue may be involved in the degradation of ingested dsRNA and hence interfere with RNAi efficiency (Kolliopoulou \& Swevers, 2014; Wynant et al., 2014). To circumvent this issue we fed relatively high concentrations of dsRNA to larvae to generate an efficient RNAi response in the gut. Higher doses of dsRNA were shown to upregulate gene expression of Argonaute and Dicer of RNAi machinery in animals (Garbutt \& Reynolds, 2012). Zhang and colleagues successfully demonstrated silencing of the chitin synthase gene in Anopheles gambiae using chitosan/dsRNA self-assembled nanoparticles, which stabilized dsRNA molecules enhancing RNAi efficacy (Zhang et al., 2010). We also evaluated oral delivery of chitosan/dsRNA nanoparticles to both whole larvae and IPLB-LdEp GM cultured tissue cells, however, partial transcript depletion was observed only in the latter (data not shown). This insufficiency may potentially have been due to resistance in the RNA transporter mechanism such as SID-1 (Winston et al., 2002) or the clathrin-dependent uptake of dsRNA (Xiao et al., 2015) in the endocytotic machineries to chitosan/dsRNA in lepidopteran insects.

This study was aimed at understanding the functional aspects of delivering dsRNA for uptake in GM midgut for the systemic depletion of targeted genes. We found oral delivery of dsRNAs produced by two methods to yield efficient depletion of target transcripts to induce RNAi in GM larvae, with suppression of certain gene targets leading to low body mass or low fecundity. When applied on a larger scale, these specific dsRNAs have potential to be used as molecular biopesticides.

ACKNOWLEDgemENTS. We would like to thank A. Park for rearing insects and making ADs.

\section{REFERENCES}

Baigude H. \& Rana T.M. 2009: Delivery of therapeutic RNAi by nanovehicles. - ChemBioChem 10: 2449-2454.

BARTEL D.P. 2004: MicroRNAs: genomics, biogenesis, mechanism, and function. - Cell 116: 281-297.

Bernstein E., Caudy A.A., Hammond S.M. \& Hannon G.J. 2001: Role for a bidentate ribonuclease in the initiation step of RNA interference. - Nature 409: 363-366.

Cheng T., Lin P., Huang L., Wu Y., Jin S., Liu C. \& Xia Q. 2016: Genome-wide analysis of host responses to four different types 
of microorganisms in Bombyx mori (Lepidoptera: Bombycidae). - J. Insect Sci. 16: 1-11.

Clemens J.C., Worby C.A., Simonson-Leff N., Muda M., Maehama T., Hemmings B.A. \& Dixon J.E. 2000: Use of doublestranded RNA interference in Drosophila cell lines to dissect signal transduction pathways. - Proc. Natn. Acad. Sci. U.S.A. 97: 6499-6503.

Doane C.C. \& McManus M.L. 1981: The Gypsy Moth: Research toward Integrated Pest Management. USDA For. Serv. Tech. Bull. No. 1585, Washington, 777 pp.

Draz M.S., Fang B.A., Zhang P., Hu Z., Gu S., Weng K.C., Gray J.W. \& CHEN F.F. 2014: Nanoparticle-mediated systemic delivery of siRNA for treatment of cancers and viral infections. Theranostics 4: 872-892.

Fire A., Xu S., Montgomery M.K., Kostas S.A., Driver S.E. \& Mello C.C. 1998: Potent and specific genetic interference by double-stranded RNA in Caenorhabditis elegans. - Nature 391: 806-811.

GARBUTT J.S. \& REYNOLDS S.E. 2012: Induction of RNA interference genes by double-stranded RNA; implications for susceptibility to RNA interference. - Insect Biochem. Mol. Biol. 42: 621-628.

Garner K.J., Hiremath S., Lehtoma K. \& Valaitis A.P. 1999. Cloning and complete sequence characterization of two gypsy moth aminopeptidase-N cDNAs, including the receptor for Bacillus thuringiensis Cry1Ac toxin. - Insect Biochem. Mol. Biol. 29: 527-535.

Grishok A., Sinskey J.L. \& Sharp P.A. 2005: Transcriptional silencing of a transgene by RNAi in the soma of C. elegans. Genes Devel. 19: 683-696.

Hagedorn H.H. \& KunKel J.G. 1979: Vitellogenin and vitellin in insects. - Annu. Rev. Entomol. 24: 475-505.

HöFte H. \& Whiteley H.R. 1989: Insecticidal crystal proteins of Bacillus thuringiensis. - Microbiol. Rev. 53: 242-255.

Honeybee Genome Sequencing Consortium 2006: Insights into social insects from the genome of the honeybee Apis mellifera. - Nature 443: 931-949.

Huvenne H. \& Smagghe G. 2010: Mechanisms of dsRNA uptake in insects and potential of RNAi for pest control: a review. $-J$. Insect Physiol. 56: 227-235.

Ivashuta S., Zhang Y., Wiggins B.E., Ramaseshadri P., Segers G.C., Johnson S., Meyer S.E., Kerstetter R.A., McNulty B.C., BolognesI R. \& HeCK G.R. 2015: Environmental RNAi in herbivorous insects. - RNA (New York) 21: 840-850.

Kamath R.S., Martinez-Campos M., Zipperlen P., Fraser A.G. \& AHRINGER J. 2001: Effectiveness of specific RNA-mediated interference through ingested double-stranded RNA in Caenorhabditis elegans. - Genome Biol. 2(1): Research0002, 10 pp.

KenNERDELl J.R. \& CARTHEW R.W. 1998: Use of dsRNA-mediated genetic interference to demonstrate that frizzled and frizzled 2 act in the wingless pathway. - Cell 95: 1017-1026.

Ketting R.F., Fischer S.E., Bernstein E., Sijen T., Hannon G.J. \& Plasterk R.H. 2001: Dicer functions in RNA interference and in synthesis of small RNA involved in developmental timing in C. elegans. - Genes Devel. 15: 2654-2659.

Kolliopoulou A. \& Swevers L. 2014: Recent progress in RNAi research in Lepidoptera: Intracellular machinery, antiviral immune response and prospects for insect pest control. - Curr. Opin. Insect Sci. 6: 28-34.

Li X., ZHANG M. \& ZHANG H. 2011: RNA interference of four genes in adult Bactrocera dorsalis by feeding their dsRNAs. - PLoS ONE 6(3): e17788, 11 pp.

Liu R.-Y., Fioravante D., Shah S. \& Byrne J.H. 2008: cAMP response element-binding protein 1 feedback loop is necessary for consolidation of long-term synaptic facilitation in Aplysia. — J. Neurosci. 28: 1970-1976.

Livshits M.A., Amosova O.A. \& Lyubchenko Yu.L. 1990: Flexibility difference between double-stranded RNA and DNA as revealed by gel electrophoresis. - J. Biomol. Struct. Dynam. 7: 1237-1249.

MaO Y.-B., CaI W.-J., Wang J.-W., Hong G.-J., TaO X.-Y., Wang L.-J., HuANG Y.-P. \& Chen X.-Y. 2007: Silencing a cotton bollworm P450 monooxygenase gene by plant-mediated RNAi impairs larval tolerance of gossypol. - Nature Biotechnol. 25: 1307-1313.

Martin P.A. 2004: A freeze-dried diet to test pathogens of Colorado potato beetle. - Biol. Contr. 29: 109-114.

Martinez J., Patkaniowska A., Urlaub H., Lührmann R. \& Tuschl T. 2002: Single-stranded antisense siRNAs guide target RNA cleavage in RNAi. - Cell 110: 563-574.

Rajagopal R., Sivakumar S., Agrawal N., Malhotra P. \& BhatNAGAR R.K. 2002: Silencing of midgut aminopeptidase N of Spodoptera litura by double-stranded RNA establishes its role as Bacillus thuringiensis toxin receptor. - J. Biol. Chem. 277: 46849-46851.

Shah N., Dorer D.R., Moriyama E.N. \& Christensen A.C. 2012: Evolution of a large, conserved, and syntenic gene family in insects. - G3 (Bethesda) 2: 313-319.

Shu Y.H., Wang J.W., Lu K., Zhou J.L., Zhou Q. \& Zhang G.R. 2011: The first vitellogenin receptor from a Lepidopteran insect: molecular characterization, expression patterns and RNA interference analysis. - Insect Mol. Biol. 20: 61-73.

Sparks M.E. \& Gundersen-Rindal D.E. 2011: The Lymantria dispar IPLB-Ld652Y cell line transcriptome comprises diverse virus-associated transcripts. - Viruses 3: 2339-2350.

Sparks M.E., Blackburn M.B., Kuhar D. \& Gundersen-Rindal D.E. 2013: Transcriptome of the Lymantria dispar (gypsy moth) larval midgut in response to infection by Bacillus thuringiensis. - PLOS ONE 8(5): e61190, 9 pp.

Suetsugu Y., Futahashi R., Kanamori H., Kadono-Okuda K., Sasanuma S.-I., Narukawa J., AJimura M., Jouraku A., NAMIKI N., SHIMOMURA M. ET AL. 2013: Large scale full-length cDNA sequencing reveals a unique genomic landscape in a lepidopteran model insect, Bombyx mori. — G3 (Bethesda) 3: 1481-1492.

Terenius O., Papanicolaou A., Garbutt J.S., Eleftherianos I., Huvenne H., Kanginakudru S., Albrechtsen M., An C., AyMERIC J.-L., BARTHEL A. ET AL. 2011: RNA interference in Lepidoptera: an overview of successful and unsuccessful studies and implications for experimental design. - J. Insect Physiol. 57: 231-245.

Tian H., Peng H., Yao Q., Chen H., Xie Q., Tang B. \& Zhang W. 2009: Developmental control of a lepidopteran pest Spodoptera exigua by ingestion of bacteria expressing dsRNA of a non-midgut gene. - PLoS ONE 4(7): e6225, 13 pp.

Timmons L. \& Fire A. 1998: Specific interference by ingested dsRNA. - Nature 395: 854.

Timmons L., COURT D.L. \& Fire A. 2001: Ingestion of bacterially expressed dsRNAs can produce specific and potent genetic interference in Caenorhabditis elegans. — Gene 263: 103-112.

Turner C.T., Davy M.W., MacDiarmid R.M., Plummer K.M., Birch N.P. \& Newcomb R.D. 2006: RNA interference in the light brown apple moth, Epiphyas postvittana (Walker) induced by double-stranded RNA feeding. - Insect Mol. Biol. 15: 383-391.

Wang K., Peng Y., Pu J., Fu W., Wang J. \& Han Z. 2016: Variation in RNAi efficacy among insect species is attributable to dsRNA degradation in vivo. - Insect Biochem. Mol. Biol. 77: $1-9$. 
Winston W.M., Molodowitch C. \& Hunter C.P. 2002: Systemic RNAi in C. elegans requires the putative transmembrane protein SID-1. - Science (New York) 295: 2456-2459.

Wynant N., Santos D., Verdonck R., Spit J., Van Wielendaele P. \& VANDEN BRoECK J. 2014: Identification, functional characterization and phylogenetic analysis of double stranded RNA degrading enzymes present in the gut of the desert locust, Schistocerca gregaria. - Insect Biochem. Mol. Biol. 46: 1-8.

Xiao D., Gao X., Xu J., Liang X., Li Q., Yao J. \& Zhu K.Y. 2015: Clathrin-dependent endocytosis plays a predominant role in cellular uptake of double-stranded RNA in the red flour beetle. — Insect Biochem. Mol. Biol. 60: 68-77.

Yoon J.-S., Shukla J.N., Gong Z.J., Mogilicherla K. \& Pall S.R. 2016: RNA interference in the Colorado potato beetle,

Leptinotarsa decemlineata: Identification of key contributors. - Insect Biochem. Mol. Biol. 78: 78-88.

Yu N., Christiaens O., Liu J., Niu J., Cappelle K., Caccia S., HuvenNe H. \& SMAgGhe G. 2012: Delivery of dsRNA for RNAi in insects: an overview and future directions. - Insect Sci. 20: 4-14.

ZAMORE P.D. 2001: RNA interference: listening to the sound of silence. - Nature Struct. Biol. 8: 746-750.

ZhaNG X., ZHANG J. \& ZHU K.Y. 2010: Chitosan/double-stranded RNA nanoparticle-mediated RNA interference to silence chitin synthase genes through larval feeding in the African malaria mosquito (Anopheles gambiae). — Insect Mol. Biol. 19: 683-693.

Received December 20, 2016; revised and accepted March 10, 2017 Published online April 5, 2017

Table S1. Genes selected for RNAi in L. dispar.

\begin{tabular}{|c|c|c|c|}
\hline $\begin{array}{l}\text { Gene } \\
\text { No. }\end{array}$ & Locus No. & $\begin{array}{l}\text { DNA Size } \\
\quad(\mathrm{bp})\end{array}$ & Gene name or product \\
\hline 1 & Locus 3 & 500 & gb|AAL26894.1| aminopeptidase N3 [Lymantria dispar] \\
\hline 2 & Locus 22 & 500 & gb|AAD31183.1| aminopeptidase N1 [Lymantria dispar] \\
\hline 3 & Locus 536 & 500 & gb|AAL26896.1| cadherin-related midgut membrane protein [Lymantria dispar] \\
\hline 4 & Locus 4003 & 501 & ref|XP_011561393.1| uncharacterized protein [Plutella xylostella] \\
\hline 5 & Locus 5421 & 450 & gb|KOB̈69102.1| Osiris 9C [Operophtera brumata] \\
\hline 6 & Locus 1129 & 500 & ref|NP_001129361.1| Osiris 18 [Bombyx mori] \\
\hline 7 & Locus 4413 & 450 & $\begin{array}{l}\text { ref|XP_001623928.1| predicted protein [Nematostella vectensis] (with partial homology } \\
\text { to gb|ABS88989.1| vitellogenin [Rhipicephalus microplus]) }\end{array}$ \\
\hline 8 & Locus 365 & 208 & gb|JAT83162.1| hypothetical protein g.17602 [Pectinophora gossypiella] \\
\hline 9 & Locus 27440 & 500 & ref|XP_012544894.1| neutral and basic amino acid transport protein rBAT isoform X2 [Bombyx mori] \\
\hline 10 & Locus 28365 & 250 & Locus_28365_Transcript \\
\hline
\end{tabular}

Table S2. Primers used in this study.

\begin{tabular}{|c|c|c|c|}
\hline $\begin{array}{c}\text { Primer } \\
\text { No. }\end{array}$ & Gene & Primer(s) & Sequence \\
\hline \multicolumn{4}{|c|}{ PCR Primers } \\
\hline 1 & Aminopeptidase N1 & LD locus 22 Ampep N1-F & ATGCATATGCGTGCAAGGT \\
\hline 2 & Aminopeptidase N1 & LD locus 22 Ampep N1-R & CGTACATGTCATCACGCAGA \\
\hline 3 & LD locus 365 & LD locus 365 M1-F & TTTTTGGAAGTAAAACCACGA \\
\hline 4 & LD locus 365 & LD locus $365 \mathrm{M} 1-\mathrm{R}$ & TCTTAACTСТACCACСТTCCAAAGA \\
\hline 5 & LD locus 28365 & LD locus 28365 M1-F & TTTACATAAATACAGCCACATTGGT \\
\hline 6 & LD locus 28365 & LD locus 28365 M1-R & AGCAGATTTGCATTAAGAATCG \\
\hline 7 & Osiris 9 & LD locus 5421 Osiris9-F2 & TGGATCTGCAAGATCAGCTC \\
\hline 8 & Osiris 9 & LD locus 5421 Osiris9-R2 & CTAAGTCCGCGCCATCACT \\
\hline 9 & Locus 4413 & LD locus $4413-F$ & CACGCCTTGGAATCGTATAAA \\
\hline 10 & Locus 4413 & LD locus $4413-R$ & GTCGGATAACCCGTAATCGT \\
\hline \multicolumn{4}{|c|}{ Primers for in vitro transcription of dsRNA } \\
\hline$\overline{11}$ & Aminopeptidase N1 & T7 LD locus 22 Ampep N1-F & GAATTAATACGACTCACTATAGGGAGAATGCATATGCGTGCAAGGT \\
\hline 12 & Aminopeptidase N1 & T7 LD locus 22 Ampep N1-R & GAATTAATACGACTCACTATAGGGAGACGTACATGTCATCACGCAGA \\
\hline 13 & LD locus 365 & T7 LD locus $365 \mathrm{M} 1-\mathrm{F}$ & GAATTAATACGACTCACTATAGGGAGATTTTTGGAAGTAAAACCACGA \\
\hline 14 & LD locus 365 & T7 LD locus 365 M1-R & GAATTAATACGACTCACTATAGGGAGATCTTAACTCTACCACCTTCCAAAGA \\
\hline 15 & LD locus 28365 & T7 LD locus $28365 \mathrm{M} 1-\mathrm{F}$ & GAATTAATACGACTCACTATAGGGAGATTTACATAAATACAGCCACATTGGT \\
\hline 16 & LD locus 28365 & T7 LD locus $28365 \mathrm{M} 1-\mathrm{R}$ & GAATTAATACGACTCACTATAGGGAGAAGCAGATTTGCATTAAGAATCG \\
\hline 17 & Osiris 9 & T7 LD locus 5421 Osiris9-F2 & GAATTAATACGACTCACTATAGGGAGATGGATCTGCAAGATCAGCTC \\
\hline 18 & Osiris 9 & T7 LD locus 5421 Osiris9-R2 & GAATTAATACGACTCACTATAGGGAGACTAAGTCCGCGCCATCACT \\
\hline 19 & Locus 4413 & T7 LD locus $4413-\mathrm{F}$ & GAATTAATACGACTCACTATAGGGAGACACGCCTTGGAATCGTATAAA \\
\hline 20 & Locus 4413 & T7 LD locus $4413-R$ & GAATTAATACGACTCACTATAGGGAGAGTCGGATAACCCGTAATCGT \\
\hline 21 & LacZ & T7 LacZ RNAi F & GAATTAATACGACTCACTATAGGGAGATGAAAGCTGGCTACAGGA \\
\hline 22 & LacZ & T7 LacZ RNAi R & GAATTAATACGACTCACTATAGGGAGAGCAGGCTTCTGCTTCAAT \\
\hline \multicolumn{4}{|c|}{ qPCR primers } \\
\hline 23 & Aminopeptidase N1 & qPCR LD locus 22 473F & CAACTCCTCAATACTATACTCTCACAATCG \\
\hline 24 & Aminopeptidase N1 & qPCR LD locus 22 573R & TCAGTAGGATTGTTCCTGAACCAA \\
\hline 25 & LD locus 365 & qPCR LD locus 365 140F & CTCTGTAGCTATATCTGGTCACACTGAA \\
\hline 26 & LD locus 365 & qPCR LD locus 365 236R & TCTCTTAACTCTACCACCTTCCAAAGA \\
\hline 27 & LD locus 28365 & qPCR LD locus 28365 25F & AGCCACATTGGTTGGACCAT \\
\hline 28 & LD locus 28365 & qPCR LD locus 28365 139R & GCGTAGTAGTTTCATATGTGTCCTTGTA \\
\hline 29 & Osiris 9 & qPCR LD locus 5421P2 102F & TCCTGAAGGACTTGGATATCTTTGA \\
\hline 30 & Osiris 9 & qPCR LD locus 5421P2 201R & GTCTGAGGATCATTGGCTAAAGGT \\
\hline 31 & Locus 4413 & qPCR LD locus $44131 \mathrm{~F}$ & CGAAAAAATCCACCATTACTTTCA \\
\hline 32 & Locus 4413 & qPCR LD locus 4413 126R & TTCAGGAGAATGTTGCTACCACTT \\
\hline 33 & 18S L. dispar & LD 18S-F & GTCTCGCAGCCGTATTAAGGCGA \\
\hline 34 & 18S L. dispar & LD 18S-R & GCACTCATCCCATCACTGGTCAGA \\
\hline
\end{tabular}


Table S3. Statistical analysis.

A. Fig. 1B Statistical significance ( $P$-values) of RNAi and body mass comparison for Control vs RNAi and L4440 vs RNAi. All statistical analysis was performed using Fisher's LSD post hoc test.

\begin{tabular}{lccccc}
\hline \multirow{2}{*}{ dsRNA } & \multicolumn{2}{c}{ Control } & & \multicolumn{2}{c}{ L4440 } \\
\cline { 2 - 3 } \cline { 5 - 6 } & 13 days & 18 days & & 13 days & 18 days \\
\hline APN1 & 0.5048 & 0.1902 & & 0.6617 & 0.3983 \\
Locus 365 & 0.0125 & 0.0195 & & 0.0429 & 0.0294 \\
Locus 28365 & 0.0236 & 0.0124 & & 0.0819 & 0.017 \\
Osi9 & 0.7124 & 0.1213 & & 0.9066 & 0.2473 \\
Locus 4413 & 0.2454 & 0.6305 & & 0.6635 & 0.8319 \\
\hline
\end{tabular}

B. Fig. 2E Statistical significance ( $P$-values) of RNAi and body mass comparison for Control vs RNAi and Mock vs RNAi. All statistical analysis was performed using Fisher's LSD post hoc test.

\begin{tabular}{lccccc}
\hline \multirow{2}{*}{ dsRNA } & \multicolumn{2}{c}{ Control } & & \multicolumn{2}{c}{ Mock } \\
\cline { 2 - 3 } \cline { 5 - 6 } & 5 days & 15 days & & 5 days & 15 days \\
\hline APN1 & 0.0039 & 0.0007 & & 0.2245 & 0.4351 \\
Locus 365 & 0.0025 & 0.0156 & & 0.1618 & 0.4218 \\
Locus 28365 & 0.002 & 0.0002 & & 0.1362 & 0.196 \\
Loci 365+28365 & 0.0006 & $<0.0001$ & & 0.0454 & 0.0867 \\
Osi9 & 0.0062 & 0.0004 & & 0.3168 & 0.2833 \\
Locus 4413 & 0.018 & 0.0006 & & 0.615 & 0.4071 \\
\hline
\end{tabular}

